\title{
Isotope production and target preparation for nuclear astrophysics data
}

\author{
Dorothea Schumann, Rugard Dressler, and Emilio Andrea Maugeri, and Stephan Heinitz \\ Laboratory of Radiochemistry, Nuclear Energy and Safety Research Department, Paul Scherrer Institute, 5232 Villigen PSI, Switzerland
}

\begin{abstract}
Targets are in many cases an indispensable ingredient for successful experiments aimed to produce nuclear data. With the recently observed shift to study nuclear reactions on radioactive targets, this task can become extremely challenging. Concerted actions of a certain number of laboratories able to produce isotopes and manufacture radioactive targets are urgently needed. We present here some examples of successful isotope and target production at PSI, in particular the production of ${ }^{60} \mathrm{Fe}$ samples used for half-life measurements and neutron capture cross section experiments, the chemical processing and fabrication of lanthanide targets for capture cross section experiments at $\mathrm{n}_{-} \mathrm{TOF}$ (European Organization for Nuclear Research (CERN), Switzerland) as well as the recently performed manufacturing of highly-radioactive ${ }^{7} \mathrm{Be}$ targets for the measurement of the ${ }^{7} \mathrm{Be}(\mathrm{n}, \alpha)^{4} \mathrm{He}$ cross section in the energy range of interest for the Big-Bang nucleosynthesis contributing to the solving of the cosmological Li-problem. The two future projects: "Determination of the half-life and experiments on neutron capture cross sections of ${ }^{53} \mathrm{Mn}$ " and " ${ }^{32} \mathrm{Si}-\mathrm{a}$ new chronometer for nuclear dating" are briefly described. Moreover, we propose to work on the establishment of a dedicated network on isotope and target producing laboratories.
\end{abstract}

\section{Introduction}

Over the past 20 years, we observe an increasing demand on precise, accurate and reliable experimentally determined nuclear data in a certain number of scientific research fields like nuclear astrophysics, basic nuclear physics, nuclear medicine, geoscience, super heavy element research and many others. This demand results likewise in an increasing number of requests for high-quality sample material in sufficient amounts and well-characterized isotopic composition as well as the manufacturing of targets in a shape suitable for the special envisaged application. The desired samples often represent or contain rare radioactive isotopes, which are very exotic and available in limited amounts only. In dependence on their activity and/or dose rate, the handling of such samples can be extremely challenging. Thus, only a handful of laboratories world-wide are allowed and capable to work with this kind of material. Correspondingly high is the waiting list for sample and target preparation, even if the isotope itself is available in sufficient amounts. To effectively exploit the very limited resources, a new level of collaboration between the target laboratories in Europe and also world-wide is urgently needed. A dedicated workshop on target preparation was performed in November 2015 at Paul-Scherrer-Institute (PSI) [1], addressing this important issue.

In the present contribution, we want to illuminate this essential problem, but also show some highlights from the PSI isotope and target production activities, beginning with a brief summary of successful experiments and mentioning at the end some future projects.

\section{Requirements for radioactive target production}

\section{Availability.}

The wanted sample material shall be available in sufficient amount.

Radioactive isotopes can be produced using nuclear reactors, neutron spallation sources or particle accelerators. In some special cases, radionuclides can be purchased from industrial companies. However, the majority of radioactive samples have to be dedicatedly produced. These methods require access to large-scale facilities and are extremely cost-intensive.

\section{Purity.}

The sample shall not contain unwanted by-products, elements and/or isotopes.

Even if a sample can be purchased from a commercial company, the chemical composition and the delivered form do in many cases not fit to the envisaged experiment. Additional purification procedures and/or transformations are necessary. For isotopes, which were produced dedicated to an experiment, as a first step the extraction from the matrix material is necessary, followed by several special purification procedures. The applied procedures shall not involve chemicals which later on can disturb the measurement. Due to the high dose rates of the radioactive samples, radioprotection concerns often require special measures, for instance hotcells and remotecontrolled handling. In a certain number of cases, also stable isotopes of the same element are hampering a 
successful experiment. These isotopes cannot be separated by chemical means, but mass separation methods have to be applied.

\section{Suitability.}

The target shall be in a shape and form suitable for the experiment.

After preparing the sample material, the target shape and form should also meet the requirements of the planned experiment. A number of issues have to be addressed, for example: i) Dimensions fitting to the installations of the facility ii) Is a target on a backing required? Which thickness shall the backing have? Which backing material is required? iii) Shall the sample be covered, or must it be an open source? Methods used to meet these requirements are for instance rolling or punching [2], electrodeposition and molecular plating, evaporation, sputtering, and others. It has to be noticed, that - in dependence on the nuclear properties of the radioisotope - for these procedures huge efforts concerning radioprotection can be necessary.

\section{Characterization.}

The target composition shall be known.

For correct interpretation of the experimental data, the knowledge of the exact composition of the target is mandatory. This includes the isotopic composition of the primary material used for target production as well as a detailed characterization of the deposited target layer and the backing. Applied methods are for instance $\alpha-, \beta$ - and $\gamma$-spectrometry as well as Inductively-Coupled Plasma Mass Spectrometry (ICP-MS) or Inductively-Coupled Plasma Optical Emission Spectrometry (ICP-OES) for measuring the atomic composition. Layer morphology can be investigated using Scanning Electron Microscopy (SEM) and Atomic-Force Microscopy (AFM). A overview on the methods can be found in [2]. Measurements of the thicknesses of both the sample material and the backing as well as the evaluation of the homogeneity are also mandatory. Also here, the radioprotection issue often complicates the procedures. Although ICP-MS and ICPOES are nowadays well-established common methods, only a few laboratories, equipped with such instruments, are able to handle radioactive samples.

\section{Isotope and target production possibilities at PSI}

High-energetic protons and secondary particles induce in matter the production of a big variety of radionuclides, some of them being very rare, exotic, and, in several cases, difficult to obtain by complementary reactions. Several of these isotopes are of high importance in nuclear astrophysics, and sufficient sample material for scientific experiments is urgently needed.

Highly-activated components stemming from the surroundings or parts of a high-power particle accelerator are a unique possibility to gain such valuable isotopes. The advantage of "mining" isotopes from waste materials consists in their principal availability, not requiring "extra" beam time. The challenge is their radiochemical isolation from the matrix.

For the exploitation of this valuable resource, we initiated a working program called ERAWAST initiative (Exotic Radionuclides from Accelerator Waste for Science and Technology) [3], aimed to extract urgently needed radioisotopes from suitable matrices and make them available for a broad community of researchers. ERAWAST is also attempting to improve the communication with the users in order to manufacture tailored samples for the planned experiments. Two workshops have been performed at PSI in 2006 and 2011, respectively, where experimenters had the opportunity to get in close contact with target producers to discuss their problems and requirements, look for new, innovative solutions and also propose new experiments, thus improving the quality of the samples and their experiment outcome in general.

In the following, we give an overview on the isotope resources at PSI, the methods for sample preparation as well as some of the highlights in scientific applications. We also mention two examples of prospective projects, which are currently ongoing or envisaged in the near future.

\subsection{Isotope sources}

PSI operates the Spallation Neutron Source SINQ, which is driven by the most powerful proton accelerator worldwide $(590 \mathrm{MeV}, 2.4 \mathrm{~mA})$, and is therefore best-suited as a producer of rare exotic radionuclides. In the frame of the ERAWAST initiative isotope separation from different matrices has been established at PSI within the past decade. In particular, the following sources for isotope extraction are available:

- Beam dumps, targets, shielding components (copper, lead, graphite and others)

- Samples from the SINQ Target Irradiation program STIP (stainless steels and other metals)

- SINQ cooling water

- Special positions in the SINQ target for dedicated irradiations

For further descriptions see $[4,5]$.

\subsection{Selected examples}

\subsection{1. ${ }^{60} \mathrm{Fe}$ - world-wide unique production at PSI}

Three Nature respective Science papers on the importance of this isotope concerning studies of supernovae phenomena were published this year [6-8]. This recently observed increasing attention on the radioactive isotope ${ }^{60} \mathrm{Fe}$ in nuclear astrophysics research implicates the necessity of reliable knowledge on its nuclear properties, in particular the decay scheme including the half-life and the branching ratios, as well as cross sections of key nuclear reactions leading to its production and/or destruction. Since ${ }^{60} \mathrm{Fe}$ is two mass units heavier than the heaviest stable iron isotope ${ }^{58} \mathrm{Fe}$, its production possibilities are limited. Double neutron capture on ${ }^{58} \mathrm{Fe}$ is one production path, but this cross section (still not experimentally determined!) is expected to be comparable low. For experiments requiring samples without a macro amount of stable carrier, production via spallation reactions is the only possibility.

At PSI, a considerable amount of ${ }^{60} \mathrm{Fe}$ was found during systematic analytical examinations in a proton irradiated copper beam dump [9]. The isotope-enriched part of the beam dump was drilled out and radiochemical 


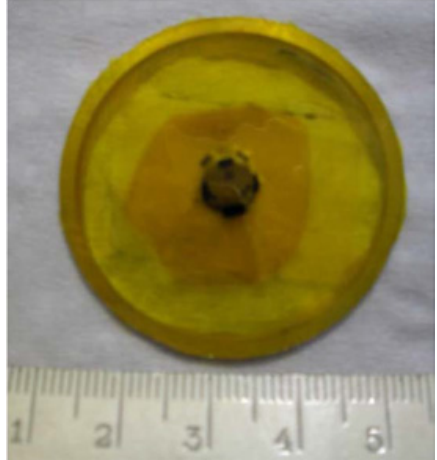

Figure 1. Photo of the final target as used in the $(\mathrm{n}, \mathrm{g})$ experiment at stellar energies (measures in cm; figure taken from [14]).

separation techniques were applied to extract about $10^{16}{ }^{60} \mathrm{Fe}$ atoms. A number of half-life measurements were performed with this material by several research groups, beginning in 2009 by Rugel et al. [10], who found a considerable disagreement in comparison to the formerly accepted value of Kutschera et al. [11], followed by Wallner et al. [12] and Ostiek [13], who confirmed the "Rugel"-value of $2.62 \cdot 10^{6}$ years. A complementary measurement at PSI is currently ongoing.

Furthermore, we produced two ${ }^{60} \mathrm{Fe}$ targets [14], which have been used for the first measurements of neutron capture cross sections at stellar [15] and thermal [16] energies. The picture (Fig. 1) shows the ${ }^{60} \mathrm{Fe}$ target which was used for the determination of the neutron capture cross section at stellar energies [15] as an example:

Standard material for Accelerator Mass Spectrometry measurements has been manufactured and is already in use at University Munich and the Australian National University (ANU) Canberra [17].

\subsection{2. ${ }^{7} \mathrm{Be}$ - the clue to the cosmological Li-problem?}

One of the most controversial and still pending problems in nuclear astrophysics is the so-called "Cosmological Lithium problem" $[18,19]$. While the standard Big-Bang nucleosynthesis $(\mathrm{BBN})$ model predicts the abundance of ${ }^{2} \mathrm{H},{ }^{3} \mathrm{He}$ and ${ }^{4} \mathrm{He}$ correctly, there is a discrepancy between the calculated ${ }^{7} \mathrm{Li}$ abundance and the one deduced from observations. In fact, the abundance of ${ }^{7} \mathrm{Li}$ observed in the atmosphere of low-metallicity stars in the Galactic halo $[20,21]$ is a factor of $2-3$ less than the value predicted by the BBN. Furthermore, new data of ${ }^{3} \mathrm{He}(\alpha, \gamma){ }^{7} \mathrm{Be}$ reaction rate and more precise values of cosmic baryon density led to a higher value of the estimated amount for the ${ }^{7} \mathrm{Li} / \mathrm{H}$ ratio increasing the discrepancy to a factor between 2.4 and 4.3 [22].

Up to now, the performance of experiments involving ${ }^{7} \mathrm{Be}$ as target material was hindered by the limited availability of the isotope and the extremely difficult handling due to the high specific activity. However, at PSI a method for filtering considerable amounts of ${ }^{7} \mathrm{Be}$ from the SINQ cooling water has been developed and successfully applied [5]. Moreover, systematic studies on target preparation using molecular plating were carried out, investigating the dependence on the most important parameters (backing material, acidity of the solution, current, voltage and duration of deposition). In Fig. 2,

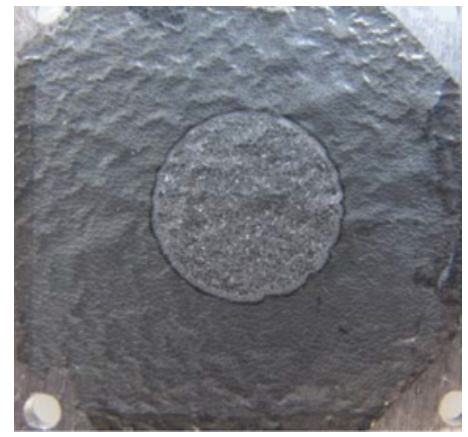

Figure 2. Picture of a ${ }^{7} \mathrm{Be}$-target on a $\mathrm{C}$ backing.

a picture of deposited $\mathrm{Be}$ on a thin graphite backing is shown as an example.

Two collection runs at SINQ were carried out in 2015 and 2016, respectively, gaining each several hundred GBq of ${ }^{7} \mathrm{Be}$. From the 2015 batch, two targets were produced for the investigation of the ${ }^{7} \mathrm{Be}(\mathrm{n}, \mathrm{a}){ }^{4} \mathrm{He}$ reaction at $\mathrm{n}_{-} \mathrm{TOF}$, one by molecular plating on an aluminum foil, the other one by evaporating droplets on a thin PE foil. The experiment has been performed with extraordinary success [23].

From the batch produced in 2016, an aliquot has been transferred to CERN, where the Isotope mass Separator On-Line facility ISOLDE was used for implanting massseparated ${ }^{7} \mathrm{Be}$ into suitable backing materials. Two targets were manufactured by this method, one for studies of the ${ }^{7} \mathrm{Be}(\mathrm{n}, \mathrm{p})^{7} \mathrm{Li}$ cross section at $\mathrm{n}_{-} \mathrm{TOF}$ and one for determination of the cross section for the reaction ${ }^{7} \mathrm{Be}(\mathrm{n}, \mathrm{a}){ }^{4} \mathrm{He}$ at thermal energies at the Institut Laue-Langevin (ILL) Grenoble. A detailed description on the manufacturing of ${ }^{7} \mathrm{Be}$ targets is given in [24].

\subsection{3. ${ }^{171} \mathrm{Tm}$ and ${ }^{147} \mathrm{Pm}$ - branching points in element synthesis}

The origin of the elements heavier than $\mathrm{Li}$ is determined by nuclear processes occurring during stellar evolution. One of these processes is the so-called $s$-process, where elements heavier than $\mathrm{Fe}$ are produced by a series of neutron capture and $\beta$-decay close to the stability line. In case of isotopes, where neutron capture and $\beta$-decay competes due to a comparable long half-life, a branching towards a higher or lower proton number becomes possible. These isotopes are called branching points. A list of 21 branching point isotopes, where a measurement of the neutron capture cross section is of great significance in the near future, has been published recently [25].

Since all branching point isotopes are radioactive, their production, handling and measurement are challenging.

For the manufacturing of targets consisting of ${ }^{171} \mathrm{Tm}$ and ${ }^{147} \mathrm{Pm}$, respectively, enriched ${ }^{170} \mathrm{Er}$ and ${ }^{146} \mathrm{Nd}$ samples were neutron irradiated in the high-flux reactor at ILL Grenoble. After the $\beta^{-}$decay of the primary neutron activation products ${ }^{171} \mathrm{Er}$ and ${ }^{147} \mathrm{Nd}$, the wanted isotopes were chemically separated using ion exchange on the strongly acidic cation exchanger AMINEX and following purification with commercially available lanthanidespecific columns. Finally, $140 \mathrm{GBq}$ of ${ }^{171} \mathrm{Tm}$ and $3 \mathrm{GBq}$ of ${ }^{147} \mathrm{Pm}$ have been deposited on two $5 \mu \mathrm{m}$ thick $\mathrm{Al}$ foils, which are positioned face to face between two $6 \mu \mathrm{m}$ thick Mylar foils (Fig. 3). Details of the target fabrication and characterization are described in [26]. The 


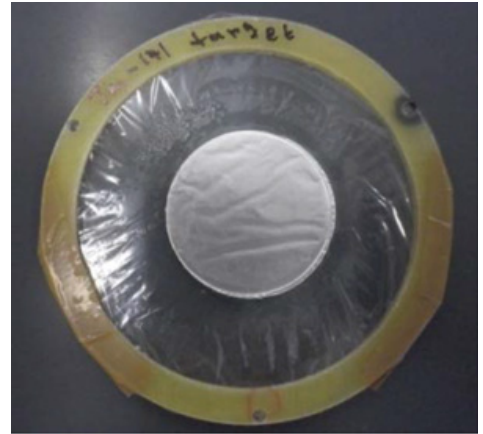

Figure 3. Picture of the ${ }^{171} \mathrm{Tm}$-target. Figure taken from [26].

two experiments on the neutron capture cross sections were successfully performed both at the Experimental Areas (EAR I and EAR II) at $n_{-}$TOF (CERN) and at the Soreq Applied Research Accelerator Facility (SARAF).

\subsection{4. ${ }^{53} \mathrm{Mn}$ - potential candidate for early solar system investigations and geochronology}

Short-lived cosmogenic radio-nuclides with half-lives of less than hundred million years $(100 \mathrm{Ma})\left(\right.$ e.g. ${ }^{10} \mathrm{Be},{ }^{26} \mathrm{Al}$, ${ }^{36} \mathrm{Cl},{ }^{53} \mathrm{Mn},{ }^{60} \mathrm{Fe},{ }^{59} \mathrm{Ni}$ ) are produced via neutron capture in explosive states of the star development or via spallogenic processes induced by high energetic protons or neutrons. Physically relevant parameters in stellar models such as neutron density, temperature, and pressure can be tested and constrained using reliable production rates in neutron capture reactions. More accurate data will be essential for the understanding of the galactic stellar evolution of successive stellar generations.

Contrary to extra-terrestrial production, the terrestrial production of cosmogenic nuclides is dominated by neutron spallation reactions; requiring knowledge of spatial and temporary variations of the neutron flux and the energy spectrum as well as reaction cross sections for neutron spallation.

Another important parameter, both for the interpretation of the galactic processes and the dating of terrestrial samples, is the half-life.

Due to the limited availability, up to now nuclear data on decay properties and cross sections are scarce. In 1960 J.P. Shedlovsky [27] revealed iron meteorites containing ${ }^{53} \mathrm{Mn}$ in an amount of about $5 \cdot 10^{11}$ atoms per $\mathrm{g}$ of iron. Using manganese extracted from such meteorites first measurements of the neutron capture cross-section at thermal neutron energy were carried out by H.T. Millard [28] in 1965 giving $\sigma_{\text {th }} \approx 170 \mathrm{~b}$ and by $R$. Wölfle et al. [29] in 1972 obtaining $\sigma_{\text {th }} \approx 66 \pm 7 \mathrm{~b}$. So far no other measurements of the neutron capture cross-section of ${ }^{53} \mathrm{Mn}$ were performed. Nuclear reaction calculations (available in nuclear data libraries as EAF-2007 [30], and TENDL2008 [31]) use the value of [29] for further predictions. In Fig. 4, known neutron capture cross-sections of ${ }^{53} \mathrm{Mn}$ are displayed together with the mean neutron energies at the UltraCold Neutrons (UCN), Beamline for neutron Optics and other Approaches (BOA), Imaging with Cold Neutrons (ICON), NEUtron Transmission RAdiography (NEUTRA) installations at PSI, the $\mathrm{n}_{-} \mathrm{TOF}$ setups at CERN, and the Frankfurt Neutron Source (FRANZ) facilities at Stern-Gerlach Center (SGC).

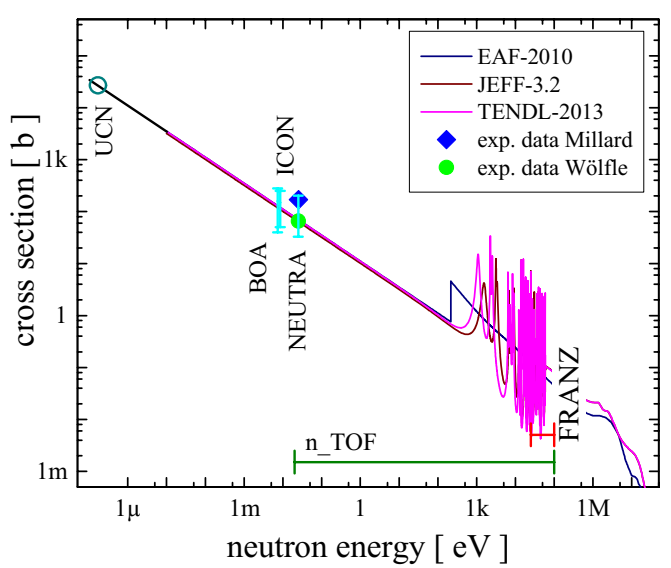

Figure 4. Comparison of known neutron capture cross-sections of ${ }^{53} \mathrm{Mn}$ with model calculations.

A few measurements have been performed to determine the half-life of this isotope. The obtained values varied from $2 \mathrm{Ma}$ to $11 \mathrm{Ma}$. Finally, the works of Honda et al. [32], Wölfle et al. [33], and Heimann et al. [34] give a consistent value of $3.8 \mathrm{Ma}$ with an uncertainty of about $10 \%$. These experiments were carried out with samples containing $2.5 \times 10^{11}$ to $1.3 \times$ $10^{13}$ atoms of ${ }^{53} \mathrm{Mn}$ limiting the precision of the measurements. However, the above discussed data are contradictory with other observations. Nyquist et al. showed in [35] that the isotopic ratio of ${ }^{53} \mathrm{Mn}$ to ${ }^{55} \mathrm{Mn}$ of different meteoritic objects is in disagreement with the deduced age using the ${ }^{206} \mathrm{~Pb}-{ }^{207} \mathrm{~Pb}$ chronometer. The obtained data suggest that the half-life of ${ }^{53} \mathrm{Mn}$ should be in the range of about $4.8 \mathrm{Ma}$, i.e. significant higher than the so far accepted value of $3.8 \mathrm{Ma}$.

Due to this unsatisfying situation, the performance of new, more sophisticated experiments with sufficient amounts of samples material is urgently needed.

Since considerable amounts of ${ }^{53} \mathrm{Mn}$ were detected in accelerator waste in the order of $10^{18}$ atoms per $g$ matrix material, we performed an extended chemical processing of proton irradiated stainless steel samples, ending up with a total amount of $10^{19}$ atoms of ${ }^{53} \mathrm{Mn}$ [36]. The manufacturing of standard material for Accelerator Mass Spectrometry (AMS) is currently ongoing [37].

We intend to start now a series of experiments determining the neutron capture cross section of ${ }^{53} \mathrm{Mn}$ using thermal-, cold-, and ultracold neutron beams available at several experimental installations at PSI as well as neutrons with a quasi-stellar energy spectrum soon available at FRANZ (SGC). A re-measurement of the halflife is also planned.

\subsection{5. ${ }^{32} \mathrm{Si}$ - a new chronometer for nuclear dating}

The predicted climate changes on Earth will have a high impact on the environment and the human society. The climate pattern observed within the past centuries leads to a better understanding of the drivers for such changes. They are the main basis for extrapolating and modelling future climate changes. More studies using new, innovative techniques are necessary to broaden the knowledge on the involved processes and accomplish a reliable scientific basis for decision makers. 
The radioactive isotope ${ }^{32} \mathrm{Si}(\mathrm{Z}=14, \mathrm{~N}=18)$ with a half-life of approximately 153 years (calculated average value taken from [38]) is a cosmogenic nuclide that is produced in the upper atmosphere by bombardment of cosmic rays on argon and then quickly transferred to the Earth surface by precipitation. It has the potential to serve as a powerful tool to understand environmental processes such as glacier dynamics, ocean and atmospheric circulation, sedimentation in lakes and oceans or groundwater flow in the recent past (300-1000 years). After the discovery of naturally existing cosmogenic ${ }^{32} \mathrm{Si}$, Lal et al. [39] proposed this nuclide as a candidate isotope for tracing and dating applications in Earth sciences. Subsequent attempts, often with heroic efforts including processing large samples, have been made to employ this nuclide for dating of old ice, e.g. by Dansgaard et al. [40]. However, for precise dating the accurate knowledge of the radionuclide"s halflife is a precondition. So far, the inconsistent database on the nuclear properties of ${ }^{32} \mathrm{Si}$ but also its extreme rareness, made its application difficult if not impossible. To overcome this, the following qualifications have to be fulfilled:

1) the ${ }^{32} \mathrm{Si}$ half-life needs to be known with uncertainty $<5 \%$;

2) reliable and reproducible routine measurement techniques including certified reference material are needed;

3) sufficient sample material for both basic and applied investigations and the routine measurements are necessary.

We propose to solve these challenges in the following way:

a) Around $200 \mathrm{MBq}$ of ${ }^{32} \mathrm{Si}$ were produced at PSI by bombarding metallic vanadium with $590 \mathrm{MeV}$ protons. A radiochemical separation system will be developed and applied for isolating and purifying ${ }^{32} \mathrm{Si}$ from the matrix material as well as from other rare exotic isotopes (e.g., ${ }^{44} \mathrm{Ti}$, $\left.{ }^{41} \mathrm{Ca},{ }^{39 / 42} \mathrm{Ar},{ }^{26} \mathrm{Al},{ }^{40} \mathrm{~K}\right)$, which are urgently needed for applications in nuclear medicine, nuclear astrophysics, environmental research, basic nuclear physics and others.

b) Measurement techniques as Liquid Scintillation Counting (LSC), Čerenkov measurement (CM), Plastic Scintillation (PS) counting, Ionization Chamber (IC) measurements, AMS and ICP-MS will be utilized and refined. The techniques developed in this course will subsequently meet the special requirements for the half-life determination of ${ }^{32} \mathrm{Si}$ as well as future routine measurements of ${ }^{32} \mathrm{Si}$. Using a fraction of the isolated ${ }^{32} \mathrm{Si}$ sample, complementary half-life measurements via different techniques will be performed.

c) Standard material for all applicable measurement techniques shall be produced and made available for routine measurements world-wide.

Establishing ${ }^{32} \mathrm{Si}$ as a new chronometer would fill the gap between the relatively short-lived ${ }^{210} \mathrm{~Pb}\left(T_{1 / 2} \approx 22 \mathrm{a}\right)$ and longer-lived ${ }^{14} \mathrm{C}\left(T_{1 / 2} \approx 5730 \mathrm{a}\right)$. The availability of ${ }^{32} \mathrm{Si}$ will foster its routine application in environmental and astrophysical applications. This has to be considered as a ground-breaking step in investigating processes in the recent past on Earth and in stars aside the commonly accepted doctrines.

\section{The need for a target preparation network}

At present, several large-scale facilities run own, dedicated target laboratories. These laboratories work mainly isolated or on a very limited network level between each other, even in the frame of pure nuclear energy research programs. Links to other research areas are missing or only little developed. Sharing of resources for isotope production and target preparation does practically not take place; coordination of requests is often left to the users. Moreover, the collaboration with the users in order to manufacture tailored samples or targets for their specific application needs urgent improvement. Owed to this situation, the development of the corresponding research infrastructures networks according to the special demands of the user communities as well as global coordination of the capabilities of the target laboratories in Europe in order to optimize the use of the very limited resources is mandatory for future ground-breaking experiments in the field of nuclear sciences and nuclear astrophysics in particular.

The global aim is to establish an overarching research infrastructure service for target production and develop a tight cooperation between the target laboratories in Europe in order to improve the production technique of wellcharacterized samples/targets. Moreover, the interaction of the target laboratories with the research teams using these targets shall be triggered and/or intensified to deliver targets best-suited for the envisaged experiments.

The following objectives are in the focus:

- Identification and promotion of synergies with other target producers

- Establishing of a network between the presently leading European infrastructures for isotope and target production

- Development of an open access data base on isotope production facilities and target manufacturers

- Coordination of targets requests, sharing of capacities, distribution of demands

- Transnational visits of scientists and especially students and postdoctoral fellows for knowledge exchange and training

- Development of new, innovative equipment for specific applications, accessible for the entire community

- Basic research on target preparation methods and characterization techniques; development of new, improved techniques

- Acquisition of new partners and, also outside Europe, to broaden the network

- Networking with the user communities.

Such a network could enable the target producers to early identify actual needs and requirements for new developments, thus triggering foresight studies for new instrumentations, methods and concepts. Dedicated projects can then be launched for concerted actions. 


\section{Conclusions and outlook}

Isotope production and radioactive target preparation are challenging tasks, requiring a tight collaboration between users and producers to obtain suitable samples, which meet the boundary conditions of the envisaged experiment. We presented here some results of such successful target production at PSI. However, the independent and isolated acting of target-producing groups is not always the best solution. A new, innovative target producer network, aimed to effectively explore the limited resources by sharing equipment and exchange knowledge, would improve the situation essentially. The bi-annual conferences of the International Nuclear Target Development Society (INTDS) [41] are a good basis for interactions of target producers. A first attempt to intensify the collaboration was started with the creation of the ANITA network (Advanced $N$ etwork for I sotope and TArget Laboratories), including 6 target producing laboratories (PSI Villigen, European Commission Joint Research Centre Directorate $\mathrm{G}$ in Geel, Belgium, Johannes Gutenberg University Mainz \& Helmholtzzentrum für Schwerionenforschung Darmstadt, Germany, Grand Accélérateur National d'Ions Lourds Caen, France and University of Warsaw, Poland). Possibilities for funding are currently explored.

\section{References}

[1] http://indico.psi.ch/conferenceDisplay . py? conf Id= 3671

[2] A. Stolarz, J. Radioanal. Nucl. Chem. 299, 913-931 (2014)

[3] D. Schumann et al., RCA 101, 501-508 (2013)

[4] D. Schumann et al., J. Phys. G 35, 014046 (2008)

[5] D. Schumann et al., RCA, 509-514 (2013)

[6] W.R. Binns et al., Science 352, 677 (2016)

[7] D. Breitschwerdt et al., Nature 532, 73 (2016)

[8] A. Wallner et al., Nature 532, 69 (2016)

[9] D. Schumann et al., RCA 97, 123-131 (2009)

[10] G. Rugel et al., PRL 103, 072502 (2009)
[11] W. Kutschera et al., NIMB 5, 430 (1984)

[12] A. Wallner et al., PRL 114, 041101 (2015)

[13] K Ostieck et al., NIMB 361, 638-642 (2015)

[14] D. Schumann et al., NIMA 613, 347 (2010)

[15] E. Uberseder et al., PRL 102, 151101 (2009)

[16] T. Heftrich et al., PRC 92, 015806 (2015)

[17] A. Wallner, personal communication

[18] R.H. Cyburt et al., PRD 69, 123519 (2004)

[19] B.D. Fields, Ann. Rev. of Nuclear and Particle Science 61, 47 (2011)

[20] F. Spite et al., Astronomy \& Astrophysics 115, 357 (1982)

[21] A. Martin et al., The Astrophysical Journal 644, 229 (2006)

[22] R.H. Cyburt et al., JCAP 11, 012 (2008)

[23] M. Barbagallo et al., PRL 117, 152701 (2016)

[24] E.A. Maugeri et al., JINST 12, PO2016 (2016)

[25] F. Kaeppeler et al., Reviews of Modern Physics 83, 157 (2011)

[26] S. Heinitz et al. RCA 2017, in press

[27] J.P. Shedlovsky, Geochim. Cosmochim. Acta 21, 156 (1960)

[28] H.T. Millard Jr, Science 147, 503 (1965)

[29] R. Wölfle, et al., Radiochim. Acta 18, 207 (1972)

[30] R.A. Forrest, et al.: UKAEA FUS 535 (2007)

[31] A.J. Koning, D. Rochman, JEFF-DOC 1262 (2008)

[32] M. Honda, M. Imamura, Phys. Rev. C 4, 1182 (1971)

[33] R. Wölfle, et al., Radiochim. Acta 18, 207 (1972)

[34] M. Heimann, et al., Geochim. Cosmochim. Acta 38, 217 (1974)

[35] L.E. Nyquist, et al., Geochim. Cosmochim. Acta 73, 5115 (2009)

[36] R. Dressler et al., Nucl. Phys.G 39, 105201 (2012)

[37] B.-A. Dittmann et al., Annual Report LCH-PSI 45 (2016)

[38] Ch. Oellet, et al., Nuclear Data Sheets for $A=32$, Nuclear Data Sheets 112, 2199 (2011)

[39] D. Lal, et al., Science 131, 332 (1960)

[40] W. Dansgaard et al., Tellus 18, 187 (1966)

[41] http://www.intds.org/ 